Research Paper

\title{
Aptavalve-gated Mesoporous Carbon Nanospheres image Cellular Mucin and provide On-demand Targeted Drug Delivery
}

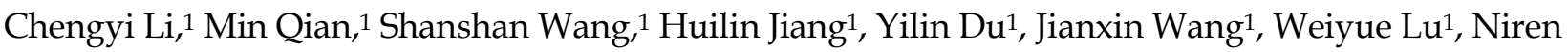 \\ Murthy ${ }^{2}$ and Rongqin Huang ${ }^{\circledR}$ \\ 1. Department of pharmaceutics, School of Pharmacy, Key Laboratory of Smart Drug Delivery, Ministry of Education, Fudan University, Shanghai 201203, P. \\ R. China. \\ 2. Department of Bioengineering, University of California, Berkeley, California 94720, USA. \\ $凶$ Corresponding author: E-mail: rqhuang@fudan.edu.cn \\ (1) Ivyspring International Publisher. This is an open access article distributed under the terms of the Creative Commons Attribution (CC BY-NC) license \\ (https:// creativecommons.org/licenses/by-nc/4.0/). See http://ivyspring.com/terms for full terms and conditions.
}

Received: 2016.12.10; Accepted: 2017.04.29; Published: 2017.07.26

\begin{abstract}
In this report, we present a mesoporous carbon nanosphere that can target drugs to tumors and image tumor biomarkers. A single-strand DNA ( $P_{0}$ aptamer) aptavalve was capped on the surface of doxorubicin-loaded oxide mesoporous carbon nanospheres (Dox-OMCN-Po) through ח-ח stacking for real-time imaging-guided on-demand targeting drug delivery. The Dox-OMCN-P could not only realize the detection of $\mathrm{MUCl}$ tumor marker with a wide linear range $(0.1-10.6$ $\mu \mathrm{mol} / \mathrm{L})$ and a low detection limit $(17.5 \mathrm{nmol})$ based on different apparatuses, but also achieve in-situ targeting imaging of cellular $\mathrm{MUCl}$ concentration in vitro and in vivo via "off-on" fluorescence biosensing. Much attractively, as a real-time feedback of the diagnostic/imaging outcomes, Dox-OMCN-P $\mathrm{P}_{0}$ accomplished the on-demand targeting drug delivery in quantitative response to $\mathrm{MUCl}$. Controllable chemotherapy with sustained release and $\mathrm{pH}$-sensitiveness, together with the potential photothermal therapy, were also clearly demonstrated. This is a simple but advanced platform, which could well achieve the real-time switchable imaging of cellular mucin for targeting cancer therapy.
\end{abstract}

Key words: MCN, aptavalve, real-time imaging, on-demand, targeting delivery

\section{Introduction}

Personalized medicine that combines imaging and targeted chemotherapy into a single nano-platform has emerged as a promising approach for cancer treatment due to the numerous advantages it offers, such as minimizing off-target toxicity, reducing multi-drug resistance, and improving the therapeutic index. ${ }^{1-3}$ In particular, if the diagnostic outcomes can be fed back to the therapeutic system in real time, to activate on-demand drug delivery, it would be a desirable platform for cancer treatment.

Cancer diagnosis based on fluorescence resonance energy transfer (FRET) biosensing has proved to be a robust technique due to the fast feedback and diffraction-limited spatial resolution of fluorescence signals. 4,5 Among those, the fluorescence quenching-recovering ("off-on") biosensors are most prevailing because of their high specificity, low background noise and strong anti-interference ability. ${ }^{6-8}$ While for drug delivery, mesoporous silica nanoparticles (MSN) are widely used owing to their unique structure, good biocompatibility and the pore gate-regularized drug release in response to a variety of tumor microenvironment stimuli.9-11 Nevertheless, the simultaneous in-situ diagnosis of tumor markers via the robust "off-on" biosensing cannot be accomplished with the drug delivery due to the optical transparency of MSN.12,13 Comparatively, oxide mesoporous carbon nanospheres $(\mathrm{OMCN})$ are 
much superior to MSN, such as the unique $\mathrm{sp}^{2}$-hybridized framework for photothermal therapy and photoacoustic imaging, ${ }^{14,15}$ and the special $\pi-\pi$ stacking with anticancer drugs for $\mathrm{pH}$-sensitive release. ${ }^{16}$ Most interestingly, it was found that OMCN could be used as a fluorescence quencher to build sensitive "off-on" aptasensor for multiple diagnosis/targeting imaging of cancers in vitro and in vivo. ${ }^{17}$ Therefore, in this work, a $\mathrm{P}_{0}$ aptavalve was gated on OMCN to construct the in-situ diagnosis and drug delivery platform, where the real-time switchable imaging of cellular mucin for on-demand targeting drug delivery can be well achieved.

In this report, the preparation of the real-time imaging-guided on-demand targeting drug delivery system and its functionalizing process were presented (Scheme 1). Firstly, OMCN was prepared, ${ }^{18}$ which had a uniform spherical morphology with a diameter of about $100 \mathrm{~nm}$ and highly ordered body-centered cubic Im $3 m$ mesostructure with a pore size of $2.8 \mathrm{~nm}$ (Fig. 1A, 1B, S1A and S1B). The anticancer drug doxorubicin (Dox), which can simultaneously act as the therapeutic agent and the fluorescence probe, was loaded into the mesopores of MCN via $\pi-\pi$ adsorption and electrostatic interaction. By optimizing the loading amount to $0.05 \mathrm{mg} / \mathrm{mg}$ (Dox/OMCN) and the loading time to $6 \mathrm{~h}$, the Dox fluorescence could be completely quenched by OMCN (Fig. S2). Consequently, a ssDNA aptamer $\left(\mathrm{P}_{0}\right)$ (Fig. S3), ${ }^{19}$ which specifically binds to the cancer cell-surface mucin1 (MUC1) marker, ${ }^{20,21}$ was used to cap the mesopores of OMCN via $\pi-\pi$ adsorption, to impede the premature escape of the drug from the mesochannels.

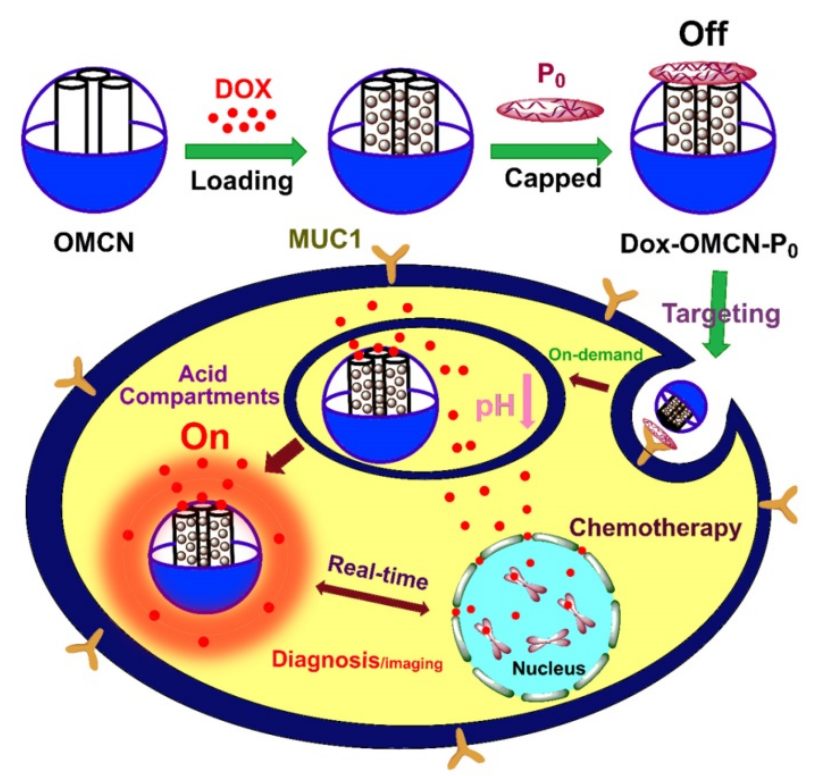

Scheme 1. Schematic preparation of OMCN-based fluorescence sensing platform and real-time switchable imaging of cellular mucin for on-demand targeting drug delivery.
As shown in Fig. 1A, S1B and S1C, the $\mathrm{P}_{0}$-capped Dox/OMCN (Dox-OMCN-P ${ }_{0}$ ) maintained its uniform spherical morphology and had a similar size as pristine OMCN. In addition, it also kept the semi-graphitic $\operatorname{Im} 3 m$ mesostructure as evidenced by the obvious peaks at $q$ values of 0.74 and $1.25 \mathrm{~nm}^{-1}$ in SAXS pattern, 22 the typical (002) and (100) reflections of graphitic carbon at $2 \theta$ values of $24.6^{\circ}$ and $40.2^{\circ}$ in XRD pattern, ${ }^{23}$ and the similar symmetry $\mathrm{A}_{1 \mathrm{~g}}$ mode and $E_{2 g}$ mode to graphitic carbon atoms at $1360 \mathrm{~cm}^{-1}$ (D-band), $1600 \mathrm{~cm}^{-1}$ (G-band) and $2933 \mathrm{~cm}^{-1}$ (2D-band) in Raman spectrum, respectively (Fig. S4). ${ }^{24}$ In contrast, some polymer-like species, composed of the aptamer $\left(\mathrm{P}_{0}\right)$ were coated on every nanospheres in Dox-OMCN-P $\mathrm{P}_{0}$ (the arrows in Fig. $1 \mathrm{C}$ and S1C), and this coating made it difficult to observe the ordered mesopores in the TEM images (Fig. S1C). In consideration of the relatively larger size of $\mathrm{P}_{0}$ than that of mesopores, it can be concluded that $\mathrm{P}_{0}$ was coated on the surfaces of OMCN to act as the gate keeper of mesopores. This distinctive coating could be further visualized by the EDX patterns and mappings, which showed the obvious $\mathrm{N}$ and $\mathrm{P}$ signals due to the surface coating of $\mathrm{P}_{0}$ (Fig. $1 \mathrm{C}-\mathrm{H}, \mathrm{S} 1 \mathrm{~A}$ and $\mathrm{S} 1 \mathrm{C}$ ). Moreover, the results of BET surface area showed an obvious decrease from $621.7 \mathrm{~m}^{2} / \mathrm{g}$ (OMCN) to 408.8 $\mathrm{m}^{2} / \mathrm{g}$ (Dox-OMCN-P $)$, which indicated the successful loading of Dox into mesopores (Fig. S5A). Besides, owing to the small defected pores (interior micropores) created in the carbon matrix via oxidation, an ongoing increase of pore size distribution ranged from 5 to $1.8 \mathrm{~nm}$ was observed (Fig. S5B and S5C). The coating amount was $1.79 \mu \mathrm{mol}$ $\mathrm{P}_{0} / \mathrm{mg}$ OMCN according to the typical UV-absorption of $\mathrm{P}_{0}$ (Fig. S6). Owing to the sealing of fluorescent anticancer drug Dox into semi-graphitic mesochannels of OMCN by $\mathrm{P}_{0}$, the constructed Dox-OMCN-P ${ }_{0}$ could act as an "off-on" fluorescence aptasensor for cancer imaging. At the same time, the diagnostic outcomes could be fed back to the therapeutic system to activate the targeting and $\mathrm{pH}$-sensitive on-demand drug delivery in real time. As shown in Fig. S7 and S8, the pH-triggered drug release (fluorescence recovery), which cooperated to the sub-acid environment in tumor, could be further regulated by the $\mathrm{P}_{0}$ cap (Fig. S9). With the increased concentration of $\mathrm{P}_{0}$ cap, a gradually decreased release of Dox accompanied with faded fluorescence recovery was observed at even $\mathrm{pH} 5.0$ (Fig. 2A). When $\mathrm{P}_{0}$ cap reached $2 \mu \mathrm{mol} / \mathrm{L}$, Dox could be well sealed into mesopores and exhibited no obvious release. Notably, the $\mathrm{P}_{0}$ cap was switchable and could be detached from OMCN via the specific binding to its receptor, MUC1 tumor maker (Fig. S10). In addition, well performances of $\mathrm{P}_{0}$ cap and MUC1 responsive Dox 
release under normal ( $\mathrm{pH}$ 7.4) and bio-mimic environment were also observed (Fig. S11-16), which further consolidated its applicability in complexed in vivo environment.

As shown in Fig. $2 \mathrm{~B}$ and $\mathrm{S10}$, the $\mathrm{P}_{0}$ gate could be gradually opened through improving the concentration of MUC1 and their incubation time, which was thereafter accompanied by the increased drug release and fluorescence recovery. Interestingly, the recovered fluorescence intensity was linear to the MUC1 concentration with a linear range of $1.06-10.6$ $\mu \mathrm{mol} / \mathrm{L}$, with a detection limit of $101 \mathrm{nmol} / \mathrm{L}$, respectively. In addition, using a more sensitive Infinite M1000 Pro microplate reader for fluorescence measurement, the detection limit was reduced to 17.5 $\mathrm{nmol} / \mathrm{L}$ and the linear range was $0.1-5.3 \mu \mathrm{mol} / \mathrm{L}$ in PBS 5.0 (Table S1 and Fig. S17). Moreover, the gate opening mediated by MUC1 was specific and the interferential proteins could not cause obvious fluorescence recovery (Fig. S18). These results suggested that Dox-OMCN-P $\mathrm{P}_{0}$ was a potential theranostic platform which could simultaneously achieve the specific imaging of MUC1 and the controllable drug release via the switchable aptavalve. Further investigations by confocal fluorescence microscopy and flow cytometric assay showed that Dox-OMCN-P ${ }_{0}$ could deliver Dox into MCF-7 cancer cells but not MCF-10A normal cells, and the delivery exhibited time-dependent behaviour (Fig. 3A, S19 and S20). This is anticipated because the MUC1 in cancer cells specifically opened the pore gates of Dox-OMCN-P, allowing the drug to be gradually released into MCF-7 cells. It is a kind of targeting drug delivery regulated by MUC1. This was confirmed by a parallel control experiment, where superfluous $\mathrm{P}_{0}(30$ $\mu \mathrm{mol} / \mathrm{L})$ was pre-incubated with MCF-7 cells before the addition of Dox-OMCN-P. No obvious fluorescence recovery was observed in different time periods (Fig. S21), which indicated the good sealing of the mesopores due to lack of enough MUC1 opener after being competitively bonded by the pre-added $\mathrm{P}_{0}$. Whereas, when different concentrations of MUC1 opener were pre-added into MCF-7 cells before being incubated with Dox-OMCN-P, a remarkable fluorescence recovery together with gradual drug release happened, and the mean fluorescence intensity was significantly positive correlation to the pre-added concentrations of MUC1, which hence suggested a potential quantitative detection of the cellular MUC1 for tumor imaging (Fig. 3B and 3C, Fig. S22). The primary MUC1 concentration on MCF-7 was calculated to be $2.33 \mu \mathrm{mol} / \mathrm{L}$ according to the fitting equation of flow cytometry. Most importantly, the drug release was also targeting and on-demand in response to the MUC1 concentration, which was therefore a personalized medicine according to the tumor progression (Fig. S23). Owing to the special delivery of Dox into MCF-7 cells, Dox-OMCN-P 0 could also be used as the therapeutic agent for cancer therapy. As indicated by the live-dead images and cytotoxicity results in Fig. 4, Dox-OMCN-P $\mathrm{P}_{0}$ exhibited a concentration-dependent cytotoxicity, which was obviously elevated on MCF-7 cells as compared to MCF-10A cells due to the targeting effect. Meanwhile, the higher cytotoxicity of $36 \mathrm{~h}$ incubation than that of $24 \mathrm{~h}$ incubation revealed that more drugs were released from the pores and entered cells via prolonged incubation time, which was in agreement with the drug release profiles (Fig. S24). Nevertheless, at both incubation time, Dox-OMCN-P $\mathrm{P}_{0}$ exhibited lower cytotoxicity than the pure Dox (Table S2), which suggested the relative sustained-release performance of Dox-OMCN-P ${ }_{0}$. Although the IC50 of Dox-OMCN-P $\mathrm{P}_{0}$ was comparatively higher due to the relatively inadequate drug, the nano-carrier of OMCN-P $\mathrm{P}_{0}$ was nontoxic (Fig. S25), by which the dose could be increased. More importantly, Dox-OMCN-P $\mathrm{P}_{0}$ could serve as the heat-producing agent for cancer photothermal therapy, ${ }^{25}$ even irradiated under a 980 nm laser in vitro and in vivo (Fig. S26, S27 and Movie S1), which might synergistically improve the outcome of chemotherapy. Therefore, Dox-OMCN-P $\mathrm{P}_{0}$ was also an excellent on-demand targeting delivery system for cancer therapy.

Further studies, including in vivo imaging and fluorescent analysis of tissue homogenate, concerning the in vivo real-time imaging-guided on-demand targeting drug delivery performances, were demonstrated (Fig. 5). The apparent fluorescence recovery and drug release were observed in tumor-bearing nude mice but not in normal nude mice via intravenous injection of switchable Dox-OMCN-P. Moreover, although intravenous injection of free Dox could also produce the fluorescent signal, there were no obvious differences between tumor-bearing and normal nude mice. And also, Dox-OMCN-P $\mathrm{P}_{0}$ injection resulted in much stronger fluorescence as compared to that of free Dox injection. These results indicated the $\mathrm{P}_{0}$-mediated targeting accumulation and the specific cap opening of Dox-OMCN-P $\mathrm{P}_{0}$ in response to MUC1-overexpressed tumor, which can therefore achieve the in vivo cancer imaging and targeting drug delivery. Notably, the ex vivo images and fluorescent intensities of tissue homogenate indicated a faster fluorescence fading in the free Dox-injected tumor-bearing mice than Dox-OMCN-P-Pinjected one, especially in the vessels-abundant tumor region. ${ }^{26}$ This might be attributed to the easy and quick diffusion of the small Dox molecules to the whole 
body through abundant blood vessels within tumors. Whereas, OMCN-P $\mathrm{P}_{0}$ nanoparticles could mediate the retention of Dox in tumors by the enhanced permeability and retention (EPR) effect. ${ }^{27}$ It is an inspiring result where Dox-OMCN-P $\mathrm{P}_{0}$ could accumulate in tumor via both active and passive targeting for real-time imaging-guided on-demand drug delivery.
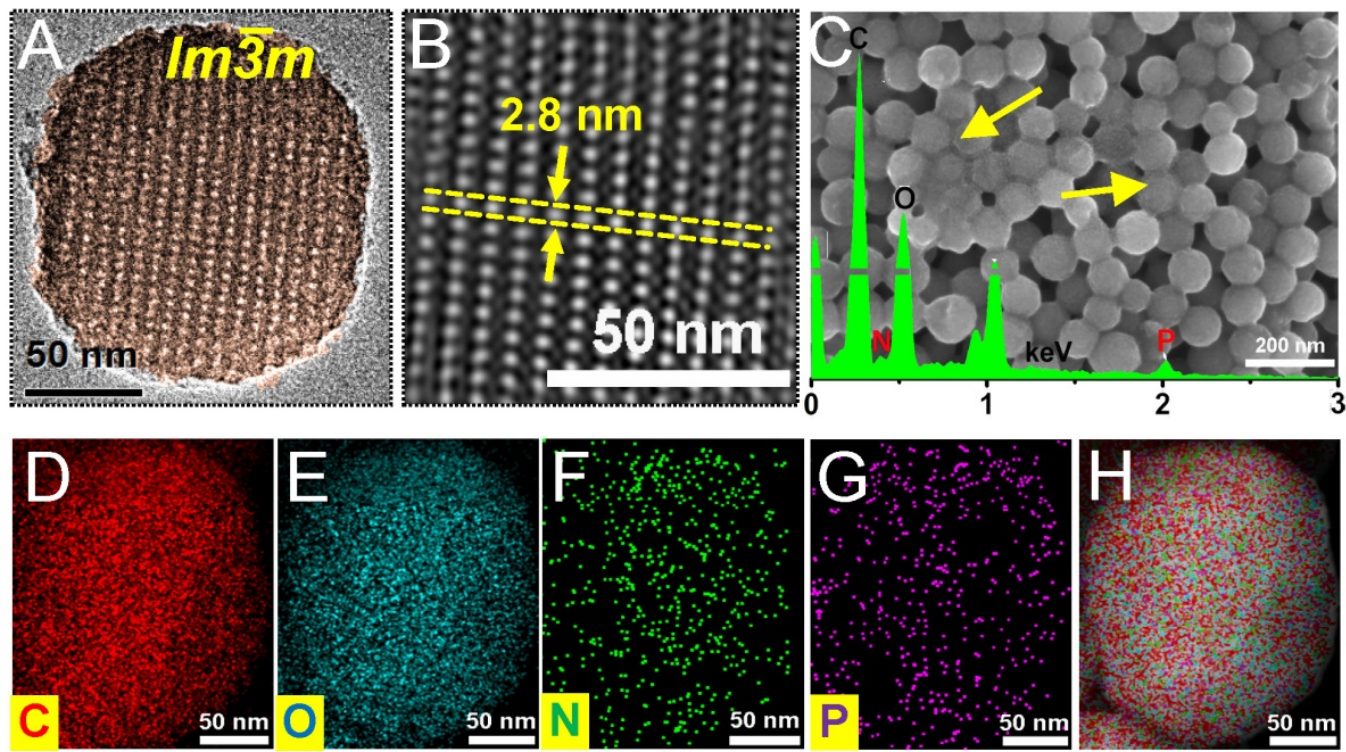

Fig 1. (A) High resolution TEM image and (B) IFFT image of OMCN. (C) SEM image and EDX pattern (inset) of Dox-OMCN-Po. (D-H) EDX mappings of carbon, oxygen, nitrogen, phosphorus and their merged image of Dox-OMCN-Po, respectively.
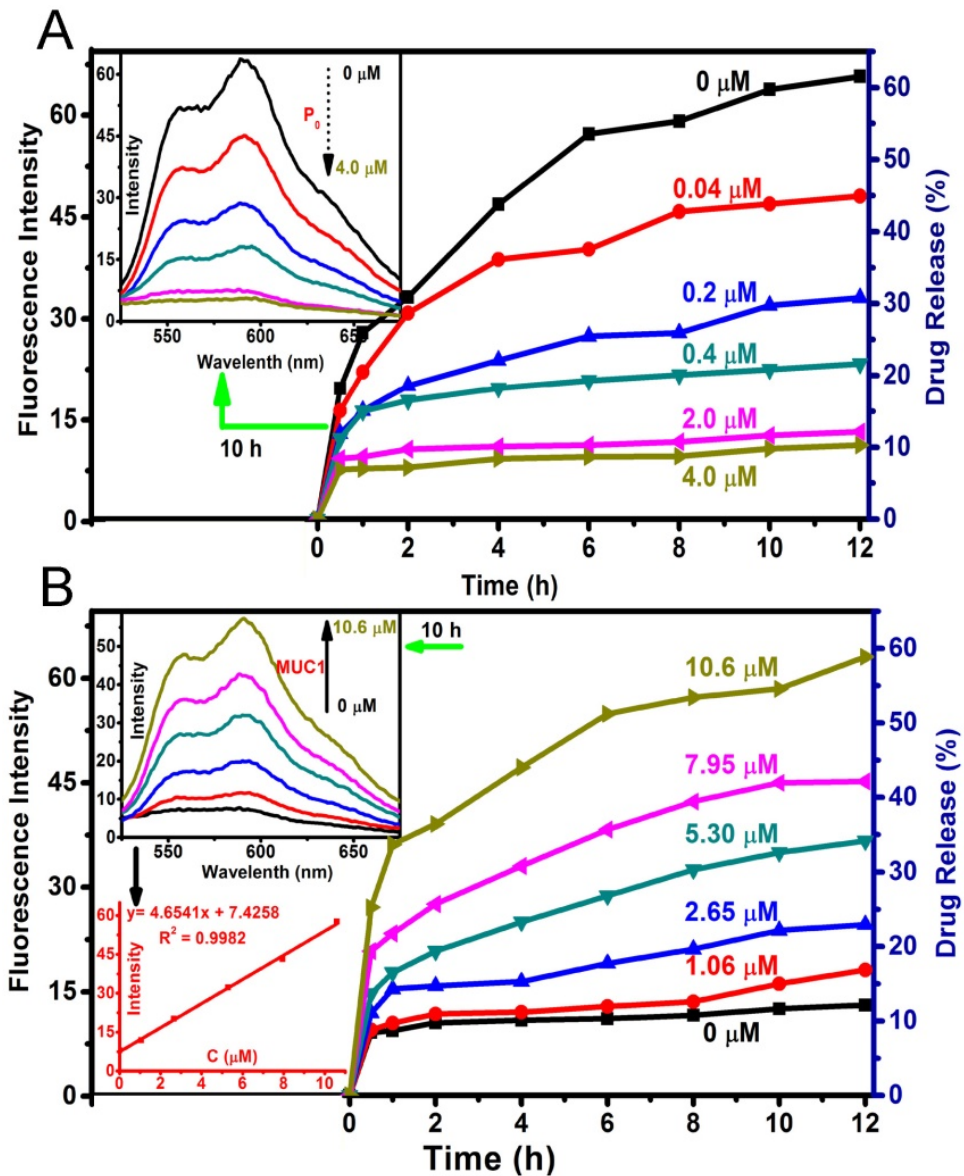

Fig 2. (A) Drug release profiles of Dox-OMCN-Po in PBS 5.0 with different concentrations of $P_{0}$ cap. The inset was the fluorescent spectra at $10 \mathrm{~h}$ release. (B) Drug release profiles of Dox-OMCN-Po in PBS 5.0 with different $\mathrm{MUCl}$ concentrations. The insets were the fluorescent spectra and the linear curve between $\mathrm{MUC1}$ concentrations and Dox fluorescence intensities after $10 \mathrm{~h}$ release. 


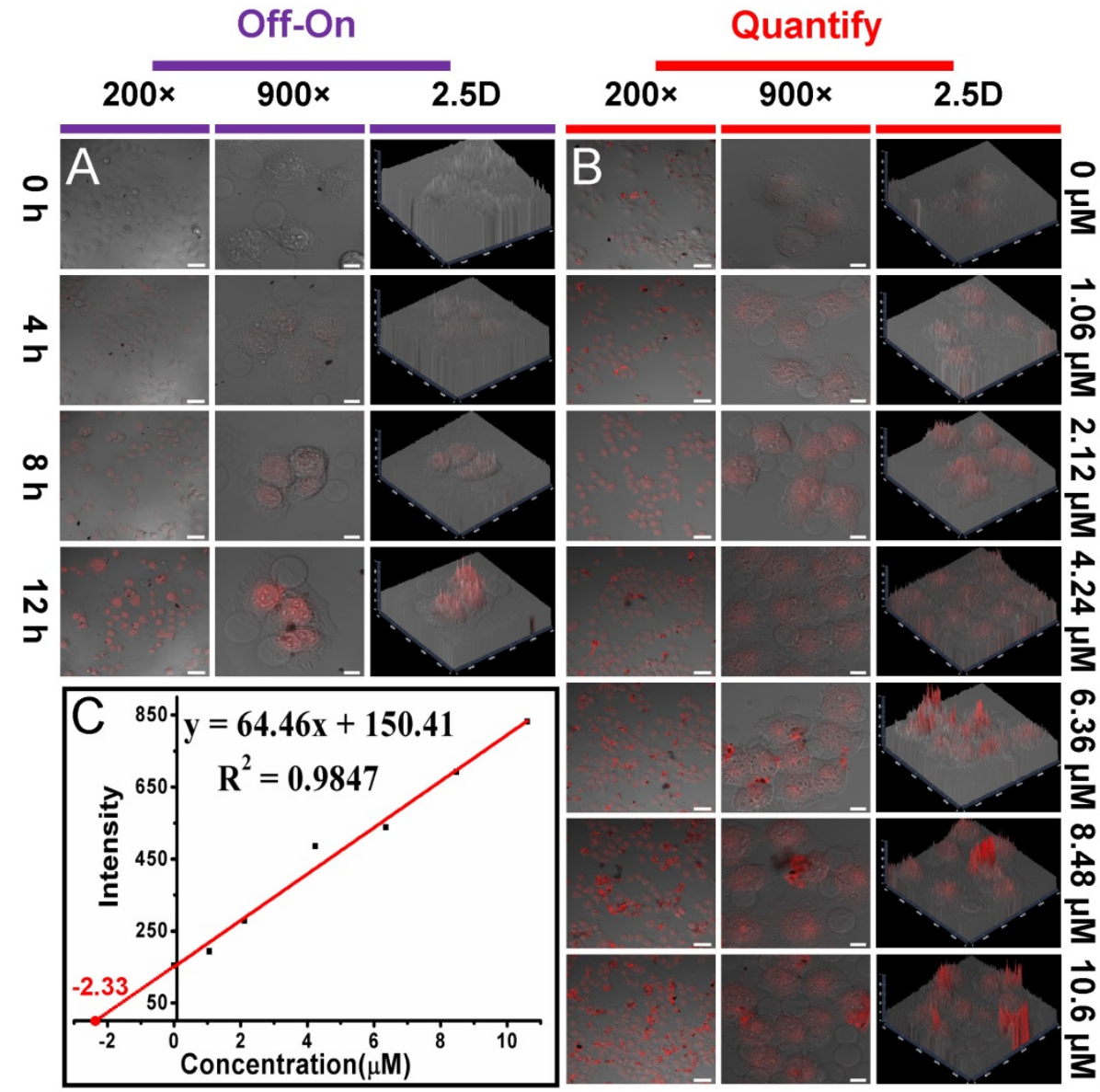

Fig 3. (A) Confocal fluorescence microscopy images of MCF-7 cells incubated with Dox-OMCN-Po for different time periods. (B) Confocal fluorescence microscopy images of MCF-7 cells incubated with Dox-OMCN-Po for $10 \mathrm{~h}$ after pre-adding different concentrations of MUCl. Scale bar: $50 \mu \mathrm{m}(200 \times)$, $10 \mu \mathrm{m}(900 \times)$. (C) Linear curve of the standard addition method for $\mathrm{MUCl}$ detection by flow cytometry analysis.

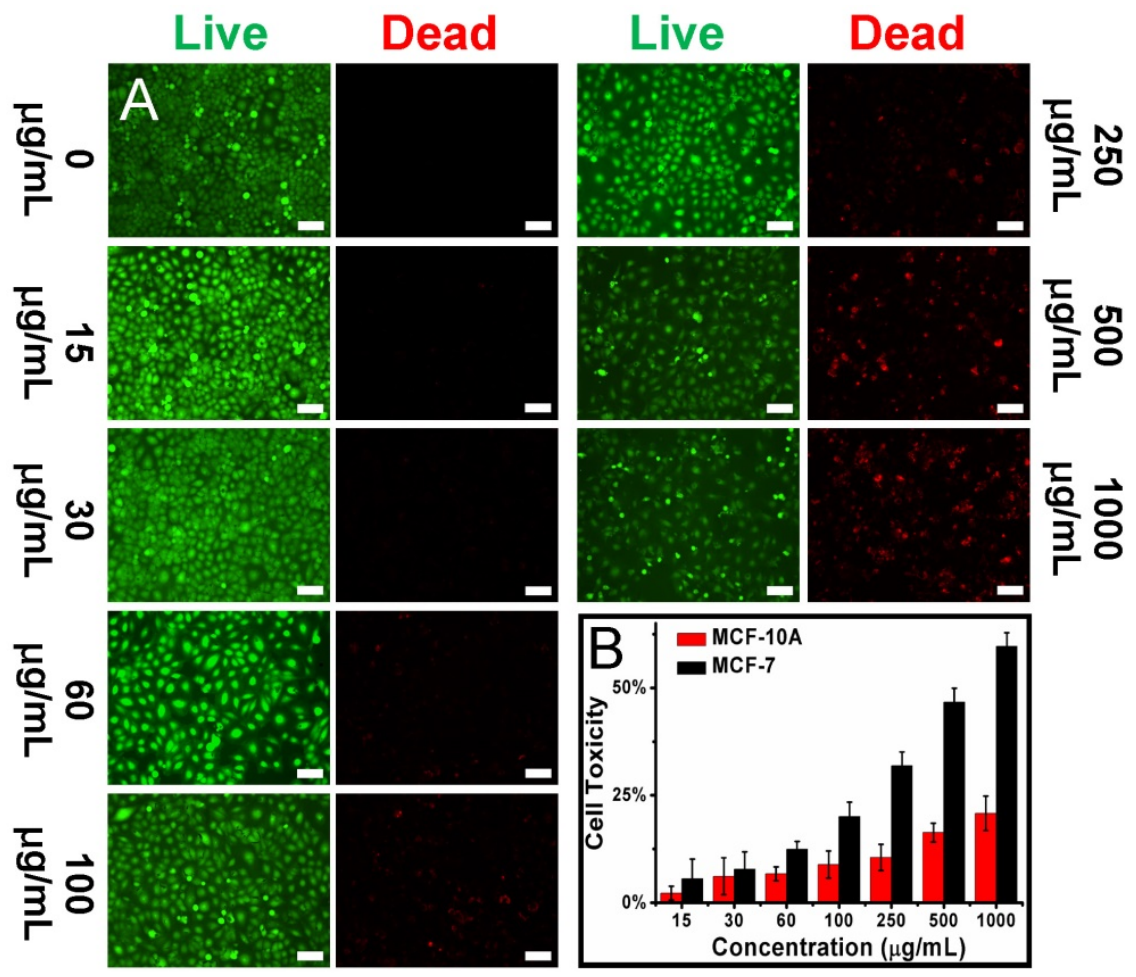

Fig 4. (A) Live-dead images of MCF-7 cells incubated with different concentrations of Dox-OMCN-Po for $24 \mathrm{~h}$. Scale bar: $150 \mu \mathrm{m}$. (B) Cell toxicity of MCF-7 cells and MCF-10A cells incubated with different concentrations of Dox-OMCN-Po for 24 h. Data are represented as mean \pm SD $(n=3)$. 


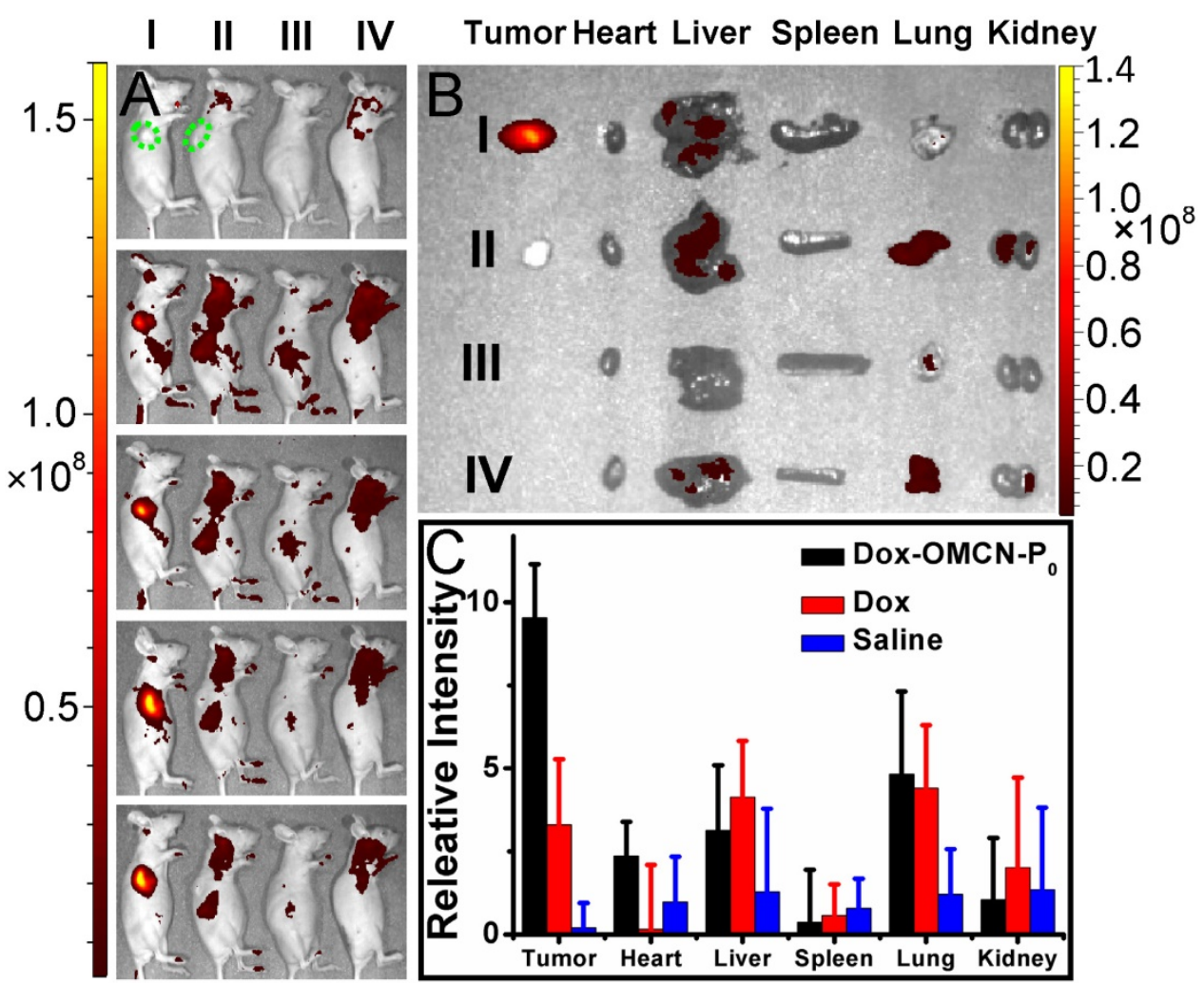

Fig 5. (A) In vivo (0, 2, 4, 8 and $12 \mathrm{~h}$, from top to bottom) and (B) ex vivo (12 h) fluorescent images of tumor-bearing (I, II) and normal (III, IV) nude mice treated with Dox-OMCN-Po (I, III) and Dox (II, IV), respectively. Green dotted lines indicated the position of tumors. (C) Fluorescent analysis of tissue homogenate of tumor-bearing mice treated with Dox-OMCN-Po, Dox and saline for $8 \mathrm{~h}$, respectively. Data are represented as mean $\pm S D(n=3)$.

\section{Conclusions}

In summary, $\mathrm{P}_{0}$ aptavalve was capped on the surface of OMCN with ordered mesopores and semi-graphitic framework to produce a real-time imaging-guided on-demand targeting drug delivery platform. This platform can realize the sensitive detection of cellular/liquid MUC1 tumor marker and the in-situ targeting imaging of cancer cells via the "off-on" fluorescence. Meanwhile, this platform can also achieve the controllable on-demand drug delivery via the switchable gate in quantitative response to MUC1. Moreover, the platform can highly accumulate in tumor and slowly release the drug with $\mathrm{pH}$-sensitiveness. Taking on these virtues and the intrinsic photothermal effect of OMCN, Dox-OMCN-P $\mathrm{P}_{0}$ is considered to be an advanced platform for real-time imaging-guided targeting cancer therapy.

\section{Supplementary Material}

Additional File 1:

Experimental Sections, supplementary figures and tables. http://www.thno.org/v07p3319s1.pdf

Additional File 2:

Movie S1. http:/ / www.thno.org/v07p3319s2.mp4

\section{Acknowledgements}

This work was supported by the grants from National Natural Science Foundation of China (81573002), National Key Basic Research Program (2013CB932502) of China (973 Program) and Sino-German Research Project (GZ995).

\section{Competing Interests}

The authors have declared that no competing interest exists.

\section{References}

1. Yang K, Feng LZ, Shi XZ, et al. Nano-graphene in biomedicine: theranostic applications. Chem Soc Rev. 2013; 42: 530-47.

2. Muthu MS, Leong DT, Mei L, et al. Nanotheranostics-Application and Further Development of Nanomedicine Strategies for Advanced Theranostics. Theronastics 2014; 4: 660-77.

3. Liu JJ, Wang C, Wang XJ, et al. Mesoporous Silica Coated Single-Walled Carbon Nanotubes as a Multifunctional Light-Responsive Platform for Cancer Combination Therapy. Adv Funct Mater. 2015; 25: 384-92.

4. Wang YG, Zhou KJ, Huang G, et al. A nanoparticle-based strategy for the imaging of a broad range of tumours by nonlinear amplification of microenvironment signals. Nat Mat. 2014; 13: 204-12.

5. Wegner KD, Hildebrandt N. Quantum dots: bright and versatile in vitro and in vivo fluorescence imaging biosensors. Chem Soc Rev. 2015; 44: 4792-834.

6. Elena Aznar, Mar O, Lluis P, et al. Gated Materials for On-Command Release of Guest Molecules. Chem Rev. 2016; 116: 561-718.

7. Zhu H, Fan JL, Wang JY, et al. An "Enhanced PET"-Based Fluorescent Probe with Ultrasensitivity for Imaging Basal and Elesclomol-Induced $\mathrm{HClO}$ in Cancer Cells. J Am Chem Soc. 2014; 136: 12820-3.

8. Li YH, Sun Y, Li JC, et al. Ultrasensitive Near-Infrared Fluorescence-Enhanced Probe for in vivo Nitroreductase Imaging. J Am Chem Soc. 2015; 137: 6407-16. 
9. Felix S, Lluis P, Mar O, et al. Gated Silica Mesoporous Materials in Sensing Applications. Chemistryopen. 2015; 4: 418-37.

10. Zhang PH, Cheng FF, Zhou R, et al. DNA-Hybrid-Gated Multifunctional Mesoporous Silica Nanocarriers for Dual-Targeted and MicroRNA-Responsive Controlled Drug Delivery. Angew Chem Int Ed. 2014; 53: 2371-5.

11. Song N, Yang YW. Molecular and supramolecular switches on mesoporous silica nanoparticles. Chem Soc Rev. 2015; 44: 3474-04.

12. Qian RC, Ding L, Ju HX. Switchable Fluorescent Imaging of Intracellular Telomerase Activity Using Telomerase-Responsive Mesoporous Silica Nanoparticle. J Am Chem Soc. 2013; 135: 13282-5.

13. Zhang ZX, Balogh D, Wang F, et al. Biocatalytic Release of an Anticancer Drug from Nucleic-Acids-Capped Mesoporous SiO2 Using DNA or Molecular Biomarkers as Triggering Stimuli. ACS Nano. 2013; 7: 8455-68.

14. Wang LM, Sun Q, Wang X, et al. Using Hollow Carbon Nanospheres as a Light-Induced Free Radical Generator To Overcome Chemotherapy Resistance. J Am Chem Soc. 2015; 137: 1947-55.

15. Biju V. Chemical modifications and bioconjugate reactions of nanomaterials for sensing, imaging, drug delivery and therapy. Chem Soc Rev. 2014; 43: 744-64.

16. Chen Y, Xu PF, Wu MY, et al. Colloidal RBC-Shaped, Hydrophilic, and Hollow Mesoporous Carbon Nanocapsules for Highly Efficient Biomedical Engineering. Adv Mater. 2014; 26: 4294-301.

17. Li CY, Meng Y, Wang SS, et al. Mesoporous Carbon Nanospheres Featured Fluorescent Aptasensor for Multiple Diagnosis of Cancer in vitro and in vivo. ACS Nano. 2015; 9: 12096-103.

18. Fang Y, Gu D, Zou Y, et al. A Low-Concentration Hydrothermal Synthesis of Biocompatible Ordered Mesoporous Carbon Nanospheres with Tunable and Uniform Size. Angew Chem Int Ed. 2010; 49: 7987-91.

19. Saghaimaroof MA, Soliman KM, Jorgensen RA, et al. Ribosomal DNA Spacer-length Polymorphisms in barley - mendelian inheritance, chromosomal location and population-dynamics. P Natl Acad Sci USA. 1984; 81: 8014-8.

20. Liu XQ, Wang F, Aizen R, et al. Graphene Oxide/Nucleic-Acid-Stabilized Silver Nanoclusters: Functional Hybrid Materials for Optical Aptamer Sensing and Multiplexed Analysis of Pathogenic DNAs. J Am Chem Soc. 2013; 135: 11832-9.

21. Kimoto $M$, Yamashige $R$, Matsunaga $K$, et al. Generation of high-affinity DNA aptamers using an expanded genetic alphabet. Nat Biotechnol. 2013; 31: 453-7.

22. Singh V, Joung D, Zhai L, et al. Graphene based materials: Past, present and future. Prog Mater Sci. 2011; 56: 1178-271.

23. Lei ZB, Lu L, Zhao XS. The electrocapacitive properties of graphene oxide reduced by urea. Energ Environ Sci. 2012; 5: 6391-9.

24. Ferrari AC, Basko DM. Raman spectroscopy as a versatile tool for studying the properties of graphene. Nat Nanotechnol. 2013; 8: 235-46.

25. Meng Y, Wang SS, Li CY, et al. Photothermal combined gene therapy achieved by polyethyleneimine-grafted oxidized mesoporous carbon nanospheres. Biomaterials. 2016; 100: 134-42.

26. Cheng L, Huang Z, Zhou WC, et al. Glioblastoma Stem Cells Generate Vascular Pericytes to Support Vessel Function and Tumor Growth. Cell. 2013; 153: 139-52.

27. Fang J, Nakamura $H$, Maeda $H$. The EPR effect: Unique features of tumor blood vessels for drug delivery, factors involved, and limitations and augmentation of the effect. Adv Drug Deliver Rev. 2011; 63: 136-51. 